\title{
Apoptosis inhibitor 5 (API-5; AAC-11; FIF) is upregulated in human carcinomas in vivo
}

\author{
LENKA KOCI $^{1}$, KATARINA CHLEBOVA ${ }^{2}$, MARTINA HYZDALOVA ${ }^{1}$, JIRINA HOFMANOVA $^{1,2}$,

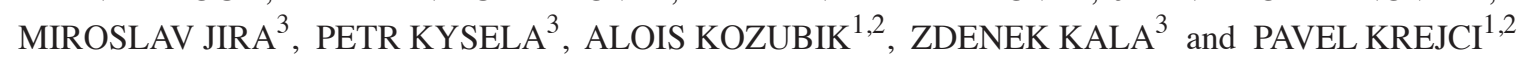 \\ ${ }^{1}$ Department of Cytokinetics, Institute of Biophysics, Academy of Sciences of the Czech Republic; \\ ${ }^{2}$ Department of Animal Physiology and Immunology, Institute of Experimental Biology, Faculty of Science, \\ Masaryk University; ${ }^{3}$ Department of Surgery, Masaryk University Hospital, Brno, Czech Republic
}

Received November 24, 2011; Accepted January 23, 2012

DOI: $10.3892 / 01.2012 .593$

\begin{abstract}
Apoptosis inhibitor 5 (API-5) is a $55 \mathrm{kDa}$ nuclear protein with potent anti-apoptotic signaling in tumor cells in vitro. In this study, we analyzed the expression of the API-5 protein in vivo in a broad spectrum of human carcinomas, including those of the colon, lung, liver, kidney, pancreas, stomach and esophagus using tumor tissues obtained during tumor resection. The results showed significant upregulation of API-5 expression in biopsies of lung $(23 \%, \mathrm{n}=13)$ and colorectal tumors $(33 \%, \mathrm{n}=27)$ in comparison with biopsies from the adjacent normal tissue. Colon cancer biopsies were used to study the cell populations with an upregulated level of expression of API-5 more closely. Using a magnetic bead-based selection for the epithelial cell marker EpCAM, we purified epithelial cells from the tumor and control tissues and analyzed these cells for API-5 expression by western immunoblotting. We observed that EpCAM-positive tumor cells expressed API-5 in all three colorectal cancer cases tested, in contrast to the control EpCAM-positive and EpCAM-negative cells isolated from the control or tumor tissues. These data suggest that the expression of the API-5 protein is upregulated in tumor epithelial cells and may serve as a prognostic marker in colorectal cancer.
\end{abstract}

\section{Introduction}

Apoptosis inhibitor 5 [API-5; FIF (fibroblast growth factor-2-interacting factor); AAC-11 (anti-apoptosis clone 11)] is a $55 \mathrm{kDa}$ nuclear protein with biological properties relevant to cancer (1). API-5 was originally identified based upon its marked anti-apoptotic action, demonstrated in mouse

Correspondence to: Dr Pavel Krejci, Department of Cytokinetics, Institute of Biophysics, Academy of Sciences of the Czech Republic, v.v.i., Kralovopolska 135, Brno CZ-612 65, Czech Republic

E-mail: krejcip@sci.muni.cz

Key words: apoptosis inhibitor 5, anti-apoptosis clone 11, carcinoma, human, apoptosis, magnetic bead selection
BALB/c3T3 fibroblasts, human cervical carcinoma CUMC-6 cells and immortalized primary liver THLE-3 cells, where ectopic API-5 expression significantly enhanced the cell survival following serum withdrawal or UV irradiation (1-4). Similarly, a decrease in API-5 levels sensitized human squamous cell carcinoma (JHU-029), colon cancer (HCT116), cervical cancer (HeLa), lung adenocarcinoma (A549), acute lymphoblastic leukemia (Molt-4), endothelial (HUVEC) and osteosarcoma (U20S) cells to a number of forms of apoptosis, demonstrating the pro-survival signaling of API-5 in several experimental tumor cell systems in vitro (4-6).

Two studies have addressed the mechanism of the API-5-mediated regulation of apoptosis. First, global Drosophila genetic screening identified API-5 as a suppressor of apoptosis mediated by E2 promoter-binding factors (E2F) in multiple cell types and developmental contexts (5). This genetic interaction was confirmed in human cancer cells in vitro and appears to be mediated by API-5 binding and inactivation of acinus, a protein necessary for apoptotic DNA fragmentation $(5,6)$. Second, API-5 protected tumor cells against the stress generated by suboptimal growth conditions or death-triggering chemicals, as demonstrated by Rigou et al, who found a marked increase of etoposide- and camptotecininduced apoptosis in various types of cancer cells caused by a depletion in the level of API-5 (6).

Taken together, the in vitro experimental evidence suggests API-5 to be an oncogene with potential therapeutic value in cancer. However, the expression of API-5 in cancer in vivo is currently poorly understood. This study was undertaken in order to determine the level of expression of the API-5 protein in human tumors in vivo in a broad spectrum of carcinomas, including those of the colon, lung, liver, kidney, pancreas, stomach and esophagus.

\section{Materials and methods}

Patient biopsies. Fresh tumor tissues were harvested during the elective scheduled tumor removal surgery, with healthy autologous tissue removed alongside the tumor used as a control. All human material was obtained at the Department of Surgery of the Masaryk University Hospital (Brno, Czech Republic), based on informed consent signed 
Table I. Summary of API-5 protein distribution in tumor and paired control tissues obtained from patients with a specific type of cancer.

\begin{tabular}{|c|c|c|c|c|}
\hline \multirow{2}{*}{$\begin{array}{l}\text { Patient } \\
\text { no. }\end{array}$} & \multirow{2}{*}{$\begin{array}{l}\text { Gender/ } \\
\text { age }\end{array}$} & \multirow[b]{2}{*}{ Diagnosis } & \multicolumn{2}{|c|}{ API-5 expression } \\
\hline & & & Control & Tumor \\
\hline 1 & $\mathrm{~F} / 48$ & Cholangiocarcinoma of the liver & - & - \\
\hline 2 & $\mathrm{M} / 48$ & Ductal adenocarcinoma of the pancreas & - & - \\
\hline 3 & $\mathrm{M} / 63$ & Adenocarcinoma of the kidney & - & + \\
\hline 4 & $\mathrm{~F} / 56$ & Spinocellular carcinoma of the esophagus & - & - \\
\hline 5 & $\mathrm{~F} / 80$ & Adenocarcinoma of the lung & - & - \\
\hline 6 & $\mathrm{M} / 54$ & Adenocarcinoma of the lung & - & - \\
\hline 7 & $\mathrm{~F} / 68$ & Adenocarcinoma of the lung & - & - \\
\hline 8 & $\mathrm{~F} / 57$ & Adenocarcinoma of the lung & - & - \\
\hline 9 & $\mathrm{M} / 63$ & Spinocellular carcinoma of the lung & - & - \\
\hline 10 & $\mathrm{M} / 71$ & Spinocellular carcinoma of the lung & - & - \\
\hline 11 & $\mathrm{M} / 61$ & Spinocellular carcinoma of the lung & - & - \\
\hline 12 & $\mathrm{M} / 76$ & Spinocellular carcinoma of the lung & - & - \\
\hline 13 & $\mathrm{~F} / 73$ & Bronchogenous adenocarcinoma of the lung & + & ++ \\
\hline 14 & $\mathrm{M} / 61$ & Adenocarcinoma of the main bronchus & - & - \\
\hline 15 & $\mathrm{~F} / 65$ & Carcinoid of the main bronchus & - & + \\
\hline 16 & $F / 81$ & Non-small cell lung cancer & - & - \\
\hline 17 & $\mathrm{M} / 47$ & Non-small cell lung cancer & + & ++ \\
\hline 18 & $\mathrm{M} / 54$ & Adenocarcinoma of the stomach & - & + \\
\hline 19 & $\mathrm{M} / 58$ & Adenocarcinoma of the stomach & - & - \\
\hline 20 & $\mathrm{M} / 72$ & Adenocarcinoma of the stomach & + & - \\
\hline 21 & $\mathrm{~F} / 75$ & Adenocarcinoma of the stomach & - & - \\
\hline 22 & $\mathrm{M} / 58$ & Adenocarcinoma of the stomach & - & - \\
\hline 23 & $\mathrm{~F} / 75$ & Spinocellular carcinoma of the stomach & + & - \\
\hline 24 & $\mathrm{~F} / 81$ & Adenocarcinoma of the coecum & - & - \\
\hline 25 & $\mathrm{M} / 58$ & Adenocarcinoma of the coecum & - & - \\
\hline 26 & $\mathrm{~F} / 75$ & Adenocarcinoma of the hepatic flexure & + & ++ \\
\hline 27 & $\mathrm{M} / 76$ & Adenocarcinoma of the hepatic flexure & - & + \\
\hline 28 & $\mathrm{M} / 75$ & Adenocarcinoma of the hepatic flexure & - & - \\
\hline 29 & $\mathrm{M} / 54$ & Adenocarcinoma of the colon transverse & - & - \\
\hline 30 & $\mathrm{~F} / 56$ & Adenocarcinoma of the colon transverse & - & - \\
\hline 31 & $\mathrm{M} / 65$ & Adenocarcinoma of the colon descending & - & + \\
\hline 32 & $\mathrm{M} / 79$ & Adenocarcinoma of the colon sigmoid & - & - \\
\hline 33 & $\mathrm{M} / 48$ & Adenocarcinoma of the rectum & + & ++ \\
\hline 34 & $\mathrm{M} / 75$ & Adenocarcinoma of the rectum & + & ++ \\
\hline 35 & $\mathrm{M} / 63$ & Adenocarcinoma of the rectum & - & - \\
\hline 36 & $\mathrm{M} / 60$ & Adenocarcinoma of the rectum & + & + \\
\hline 37 & $\mathrm{M} / 57$ & Adenocarcinoma of the rectum & - & - \\
\hline 38 & $\mathrm{M} / 87$ & Adenocarcinoma of the rectum & - & + \\
\hline 39 & $\mathrm{~F} / 71$ & Adenocarcinoma of the rectum & - & - \\
\hline 40 & $\mathrm{~F} / 77$ & Adenocarcinoma of the rectum & - & + \\
\hline 41 & $\mathrm{M} / 62$ & Adenocarcinoma of the rectum & - & - \\
\hline 42 & $\mathrm{M} / 76$ & Adenocarcinoma of the rectum & - & - \\
\hline 43 & $\mathrm{~F} / 63$ & Adenocarcinoma of the rectum & - & - \\
\hline 44 & $\mathrm{~F} / 74$ & Adenocarcinoma of the rectum & - & - \\
\hline 45 & $\mathrm{M} / 50$ & Adenocarcinoma of the rectum & - & - \\
\hline 46 & $\mathrm{M} / 62$ & Adenocarcinoma of the rectum & - & - \\
\hline 47 & $\mathrm{~F} / 53$ & Adenocarcinoma of the rectum & - & - \\
\hline 48 & $\mathrm{M} / 69$ & Adenocarcinoma of the rectum & - & + \\
\hline 49 & $\mathrm{M} / 81$ & Adenocarcinoma of the rectum & - & $(+)$ \\
\hline 50 & $\mathrm{M} / 60$ & Adenocarcinoma of the rectum & - & $(+)$ \\
\hline
\end{tabular}

Quantitative differences in expression are indicated in patients where the API-5 protein was detected in the control and tumor tissues. (+), API-5 protein detected only in EpCAM-positive tumor cells (Fig. 1B); F, female; M, male. 
A

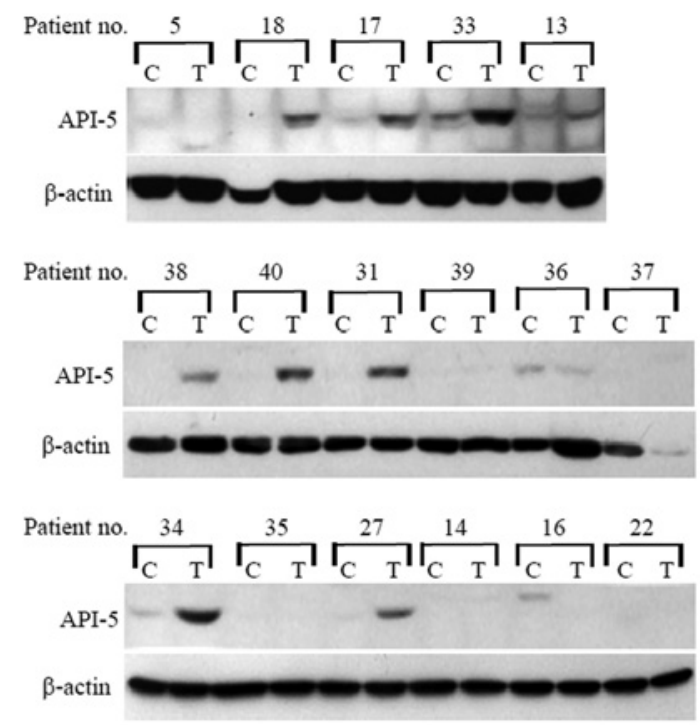

B

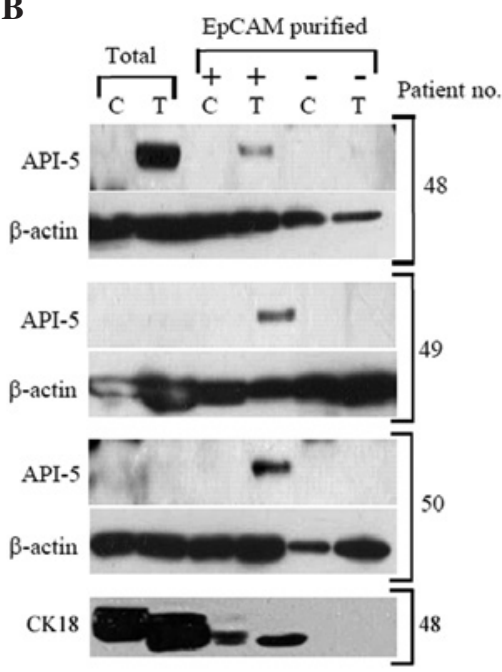

Figure 1. Total level of API-5 protein in (A) human carcinoma biopsies and (B) EpCAM-purified cells. (A) Fresh tumor tissues (T) with healthy autologous tissue removed alongside the tumor used as a control (C) of selected biopsies (numbered as in Table I). (B) Tumor tissue (T) or appropriate control (C) from total sample or EpCAM-positive (+)/negative (-) cells (patient nos. $48-50$ in Table I). $\beta$-actin was used as the loading control and cytokeratin 18 (CK18) was used as the EpCAM (epithelial cell) purification control.

by the patient and approval by the Ethics Committee of the Masaryk University Hospital. Samples were collected during surgery only when it was clear that the collection would not damage the resected tissue and affect the subsequent histological processing, pathological assessment or diagnosis. The results of this study did not affect patient management following the surgery. All of the experimental procedures were in compliance with the local laws of the Czech Republic and the Declaration of Helsinki.

Epithelial cell isolation. Tissues samples were obtained from three patients with colon cancer undergoing tumor resection (patient nos. 48-50 in Table I). Solid tissues were dissected into small fragments, incubated with collagenase type III (200 U/ml; Worthington, Lakewood, NJ, USA) and DNAseI (100 U/ml; Sigma-Aldrich, Prague, Czech Republic) to obtain enzymatic disaggregation. The tissues were then filtered with sterile $50 \mu \mathrm{m}$ nylon meshes and the resulting single cell suspensions were subjected to a magnetic bead selection to purify the EpCAM-positive epithelial cells from the tumor and paired control tissues, according to the manufacturer's instructions (Miltenyi Biotec, Auburn, CA, USA).

Western blot analysis. The cells, lysed in a Laemmli sample buffer and diluted to an equal concentration, were processed and subjected to SDS-PAGE, as previously described (5). The membranes were probed with rabbit anti-API-5 (a gift from Dr R. Rubin, Jefferson Medical College, Philadelphia, PA, USA), mouse anti-cytokeratin-18 (11-106-C100; Exbio, Vestec, Czech Republic) and mouse anti- $\beta$-actin (A5441; Sigma-Aldrich) antibodies. Proteins were visualized using the horseradish peroxidase-labelled secondary antibodies mouse anti-IgG and rabbit anti-IgG (NA934 and NA931; Amersham
Biosciences, Bucks, UK) and enhanced chemiluminescence (Millipore, Prague, Czech Republic). Equal loading was verified using $\beta$-actin.

\section{Results}

The level of expression of API-5 was significantly upregulated in certain carcinomas. We analyzed the level of API-5 protein expression in 50 tissue biopsies freshly obtained during tumor resection in patients suffering from various forms of carcinoma, including colon, lung, stomach, liver, pancreas, esophagus and kidney. With autologous tissue obtained from the morphologically normal surgical margin of the same biopsy serving as a paired control, we detected the API-5 protein in 14 of the total tumor tissues $(29 \%)$ and in 8 control samples (16.7\%; Table I). When compared with the paired controls, the level of expression of the API-5 protein was significantly upregulated in 9 colon (mostly rectum) cancer biopsies (33\%, $\mathrm{n}=27), 3$ lung cancer biopsies $(23 \%, \mathrm{n}=13)$ and one kidney biopsy $(100 \%, \mathrm{n}=1$; Fig. 1A). Within the stomach carcinoma samples, we found that the level of API-5 protein expression was elevated (compared with the paired tissues) in one tumor sample and two control samples (16.7 vs. $33 \%, n=6$; Table I).

Tumor epithelial cells expressed API-5. Three representative rectum cancer biopsies were analyzed to study the cell populations with upregulated API-5 more closely. Using a magnetic bead-based selection for the epithelial cell marker EpCAM (7), we purified epithelial cells from the tumor and control tissues and detected the level of API-5 expression in these cells. Fig. 1B shows that EpCAM-positive tumor cells expressed API-5 in all three cases, in contrast to control EpCAM-positive and EpCAM-negative cells, which were 
isolated from the control and tumor tissues. Notably, in two of the cases we detected API-5 in the EpCAM-positive tumor cells but not in the corresponding total tissue biopsy (Fig. 1B).

\section{Discussion}

Previous studies have demonstrated marked API-5 expression in human leukemia, nasopharyngeal, renal and cervical cancer cells $(2,6)$. Similarly, we found abundant expression of the API-5 protein in all nine of the tested colon epithelial cell lines derived from different stages of colon cancer development: fetal FHC, adenoma AA/C1, AA/C1/SB10 and RG/ C2, adenocarcinoma HT-29, Caco-2 and SW480, carcinoma HCT-116 and metastatic SW620 cell lines (data not shown). Thus, it appears that the abundant expression of API-5 is associated with most cancer cell lines in vitro. In vivo, the level of expression of API-5 was low in normal tissue but increased in metastatic cervical cancer tissue (2). An increased API-5 level was also detected in colonic epithelium in longstanding ulcerative colitis (8). Of 94 non-small cell lung cancer biopsies compared with paired normal lung tissues, RT-PCR analysis detected API-5 transcripts in $12.7 \%$ of the cancers and its presence was correlated with poor survival (9). Previously, we found that API-5 was markedly upregulated in the B-lymphocytes of patients suffering from B-cell chronic lymphoid leukemia, a blood malignancy characterized by defective apoptosis of mature B-lymphocytes (10).

Our analysis of patient biopsies from predominantly lung and colorectal tumor vs. normal tissue revealed an increased level of API-5 protein expression in tumors but not in the adjacent normal tissue. The results from 5 patients with stomach adenocarcinoma were unclear, as we detected an increased level of API-5 expression in two of the paired normal controls but not in the tumor tissues (Table I). We found that an increased API-5 protein expression level was particularly associated with the epithelial cells obtained from rectum cancer biopsies (Fig. 1B). Probably due to a lower percentage of the API-5-expressing epithelial cells, it was not possible to detect this protein in the total tissue sample. These data suggest that tumor epithelial cells themselves are the source of upregulated API-5 and that the true percentage of colon cancers upregulating API-5 may be higher than that found in total tissue biopsies.

In conclusion, we present in vivo evidence of upregulated API-5 protein expression in certain types of human carcinomas, particularly lung and colorectal. Moreover, we have demonstrated that epithelial cells are the prominent API-5-expressing cell type. Given the experimental anti-apoptotic signaling of API-5, further studies are required to address the role of the upregulation of API-5 in the development and progression of carcinoma.

\section{Acknowledgements}

This study was supported by the Ministry of Education, Youth and Sports of the Czech Republic (MSM0021622430; MSMT NPV II 2B06060) and the Czech Science Foundation (301/09/0587, 305/09/1526, 305/11/0752, 303/09/H048 and P301/11/1730).

\section{References}

1. Tewari M, Yu M, Ross B, Dean C, Giordano A and Rubin R: AAC-11, a novel cDNA that inhibits apoptosis after growth factor withdrawal. Cancer Res 57: 4063-4069, 1997.

2. Kim JW, Cho HS, Kim JH, et al: AAC-11 overexpression induces invasion and protects cervical cancer cells from apoptosis. Lab Invest 80: 587-594, 2000.

3. Sutherland HG, Mumford GK, Newton K, et al: Large-scale identification of mammalian proteins localized to nuclear sub-compartments. Hum Mol Genet 10: 1995-2011, 2001.

4. Wang Z, Liu H, Liu B, et al: Gene expression levels of CSNK1A1 and $\mathrm{AAC}-11$, but not NME1, in tumor tissues as prognostic factors in NSCLC patients. Med Sci Monit 16: CR357-364, 2010.

5. Morris EJ, Michaud WA, Ji JY, Moon NS, Rocco JW and Dyson NJ: Functional identification of Api5 as a suppressor of E2F-dependent apoptosis in vivo. PLoS Genet 2: e196, 2006.

6. Rigou P, Piddubnyak V, Faye A, et al: The antiapoptotic protein AAC-11 interacts with and regulates Acinus-mediated DNA fragmentation. EMBO J 28: 1576-1588, 2009.

7. Dalerba P, Dylla SJ, Park IK, et al: Phenotypic characterization of human colorectal cancer stem cells. Proc Natl Acad Sci USA 104: 10158-10163, 2007.

8. Pekow JR, Dougherty U, Mustafi R, et al: miR-143 and miR-145 are downregulated in ulcerative colitis: Putative regulators of inflammation and protooncogenes. Inflamm Bowel Dis: May 6, 2011 (E-pub ahead of print).

9. Sasaki H, Moriyama S, Yukiue H, et al: Expression of the antiapoptosis gene, AAC-11, as a prognosis marker in non-small cell lung cancer. Lung Cancer 34: 53-57, 2001.

10. Krejci P, Pejchalova K, Rosenbloom BE, et al: The antiapoptotic protein Api5 and its partner, high molecular weight FGF2, are up-regulated in B cell chronic lymphoid leukemia. J Leukoc Biol 82: 1363-1364, 2007. 